Pacific Journal of Mathematic 


\title{
BOUNDED GENERATORS OF LINEAR SPACES
}

\author{
T. ITo AND T. SEIDMAN
}

Let $S_{\varphi}=\left\{x \in X: \sup _{\alpha} \varphi_{\alpha}(x)<\infty\right\}$ where $\varphi=\left\{\varphi_{\alpha}\right\}$ is a family of semi-norms determining the topology of $X$. It is shown that $\varphi$ may be chosen so $S_{\varphi}$ is dense if and only if $\boldsymbol{X}$ has a bounded generating set if and only if there is a continuous norm on $X^{*}$. It is shown that these conditions hold for separable Fréchet spaces and for quotients of products of Banach spaces. An example is given of a Fréchet space containing no bounded generating set thus contradicting an assertion of $L$. Maté that $S_{\varphi}$ is dense for Fréchet spaces.

All spaces, $\boldsymbol{X}, \boldsymbol{Y}, \cdots$, in this paper are linear spaces with locally convex Haussdorf linear topologies. Given $X$, let $\Phi=\Phi(X)$ be the set of all families $\varphi=\left\{\varphi_{\alpha}\right\}$ of continuous semi- norms on $X$ determining the topology; for $\varphi \in \Phi$, let $S_{\varphi}=\left\{x \in X: N_{\varphi}(x)=\sup _{\varphi} \varphi_{\alpha}(x)<\infty\right\}$. For subsets $\Gamma \subset X$, let $[\Gamma]$ be the closed linear hull of $\Gamma$; if $X$ has a bounded generator, i.e., if there is a bounded set $\Gamma \subset X$ with $[\Gamma]=X$, we call $X$ a 'BG space'. Again, for a subset $\Gamma \subset X$, let $\Gamma^{\perp}$ be its annihilator, $\Gamma^{\perp}=$ $\left\{\xi \in X^{*}: \xi(x)=0\right.$ for $\left.x \in \Gamma\right\}$, let $\Gamma^{0}$ be its polar set, $\Gamma^{0}=\left\{\xi \in X^{*}:|\xi(x)| \leqq 1\right.$ for $x \in \Gamma$ \}, and let $\nu_{r}$ be the Minkowski gauge of $\Gamma^{0}$ so, for $\xi \in X^{*}, \nu_{I}(\xi)=$ $\inf \left\{t: \xi \in t \Gamma^{0}\right\}=\sup \{|\xi(x)|: x \in \Gamma\}$. By the definition of the strong topology on $X^{*}, \nu_{T}(\xi)=\inf \left\{t: \xi \in t \Gamma^{0}\right\} \sup \{|\xi(x)|: x \in \Gamma\}$. By the definition of the strong topology on $X^{*}, \nu_{\Gamma}$ is (strongly) continuous (and everywhere finite) if and only if $\Gamma$ is bounded in $X$; it is a norm if and only if $\left[\Gamma^{\prime}\right]=X$.

It is asserted in Maté [1] that, given a Fréchet space $\boldsymbol{X}$ and $\varphi \in \Phi(\boldsymbol{X}), \varphi=\left\{\varphi_{1}, \varphi_{2}, \cdots\right\}$, the set $S_{\varphi}$ is dense in $\boldsymbol{X}$. That this need not hold in general may be seen from the following elementary example.

EXAmpLE 1. Let $\boldsymbol{X}=\boldsymbol{s}$ be the space of all sequences $x=\left[x_{1}, x_{2}, \cdots\right]$ with the topology of coordinate-wise convergence. We may take $\varphi \in \Phi$ to be given by $\varphi_{n}(x)=n \max \left\{\left|x_{k}\right|: k \leqq n\right\}(n=1,2, \cdots)$ or $\psi \in \Phi$ with $\psi_{n}(x)=\left|x_{n}\right|$. Then $S_{\psi}$ is the set of all bounded sequences (which $i s$ dense in $\boldsymbol{X}$ ) but $S_{\varphi}=\{0\}$ since, for $k \geqq n$ and any $x \in X$, $\left|x_{k}\right| \leqq \varphi_{n}(x) / n \leqq N_{\varphi}(x) / n$.

This makes it clear that the density of $S_{\varphi}$ in $X$ depends on the choice of $\varphi \in \Phi$ and raises the question: Can one (in particular, if $\boldsymbol{X}$ is a Fréchet space) choose $\varphi \in \Phi(X)$ so that $S_{\varphi}$ is dense in $\boldsymbol{X}$ ? or, somewhat more generally: Can one choose $\varphi \in \Phi(X)$ so that the closure of $S_{\varphi}$ is a specified closed subspace $Y$ ? We begin by showing the 
equivalence of certain conditions on $\boldsymbol{X}, \boldsymbol{Y}$.

THEOREM 1. Given a space $\boldsymbol{X}$ and a closed subspace $\boldsymbol{Y} \cong \boldsymbol{X}$, the following conditions are equivalent:

(A) There exists $\varphi \in \Phi(X)$ such that $\bar{S}_{\varphi}=\boldsymbol{Y}$.

(B) There exists a bounded set $\Gamma \subset \boldsymbol{X}$ with $[\Gamma]=\boldsymbol{Y}$.

(C) $Y$ is a BG space.

(D) There exists a (strongly) continuous semi-norm $\hat{\nu}$ on $X^{*}$ such that $\hat{\nu}^{-1}(0)=\boldsymbol{Y}^{\perp}$.

(E) There exists a (strongly) continuous norm $\nu$ on $\boldsymbol{Y}^{*}$.

Proof. The equivalence of (B) and (C) is immediate. The equivalence of (D) and (E) follows from the observation that we may take $\hat{\nu}=\nu \circ \tau$ (where $\tau: X^{*} \rightarrow Y^{*}$ is the 'restriction of domain' map) and noting that $\tau$ is continuous, open, surjective, and linear with kernel $\boldsymbol{Y}^{\perp}$. We now show the equivalence of $(\mathrm{A})$ and $(\mathrm{B})$ and of $(\mathrm{C})$ and $(\mathrm{E})$. Given (A), set $\Gamma=\left\{x \in X: N_{\varphi}(x) \leqq 1\right\}$. Clearly $\Gamma$ is bounded; as $\Gamma$ is balanced and convex, $[\Gamma]=\bar{U}_{k} k \Gamma$ which is just $\bar{S}_{\varphi}=\boldsymbol{Y}$. Conversely, suppose we are given (B). Let $\hat{\psi}$ be a set of continuous semi-norms on $\boldsymbol{X}$ such than $\cap\left\{\left|\hat{\psi}_{\alpha}^{-1}(0)\right|: \hat{\psi}_{\alpha} \in \hat{\psi}\right\}=\boldsymbol{Y}$; such a set $\hat{\psi}$ may be obtained from any element of $\Phi(\boldsymbol{X} / \boldsymbol{Y})$. Let $A$ be the index set of $\hat{\psi}=\left\{\hat{\psi}_{a}\right\}$, which we may assume infinite and let $B$ be the set of all finite subsets of $A$; for $\beta \in B, x \in X$, set $\tilde{\psi}_{\beta}(x)=n_{\beta} \max \left\{\hat{\psi}_{\alpha}(x): \alpha \in \beta\right\}$ (where $n_{\beta}$ is the cardinality of $\beta$ ) and let $\tilde{\psi}=\left\{\tilde{\psi}_{\beta}\right\}$. Next choose any $\hat{\varphi} \in \Phi(X)$ and define $\tilde{\varphi} \in \Phi$ as follows: for $\hat{\varphi}_{r} \in \hat{\varphi}$ there is, as $\Gamma$ is bounded, $c_{\gamma}>0$ such that $\Gamma \leqq c_{\gamma}\left\{x \in \dot{X}: \hat{\varphi}_{\gamma}(x)<1\right\}$; set $\tilde{\varphi}_{\gamma}(x)=\hat{\varphi}_{\gamma}(x) / c_{\gamma}$ and $\tilde{\varphi}=\left\{\tilde{\varphi}_{\gamma}\right\}$. Finally, let $\varphi=\tilde{\psi} \cup \tilde{\varphi}$; since each $\tilde{\psi}_{\beta} \in \tilde{\psi}$ is continuous and $\widetilde{\varphi} \in \Phi$, we have $\varphi \in \Phi$. For $x \in \Gamma$ and $\varphi_{*} \in \varphi$, we have $\varphi_{*}(x)=0$ if $\varphi_{*} \in \widetilde{\psi}$ (as $\Gamma \subset Y$ ) and $\varphi_{*}(x) \leqq 1$ if $\varphi_{*} \in \widetilde{\varphi}$ so $N_{\varphi}(x) \leqq 1$. Thus $\Gamma \subset S_{\varphi}$ whence $\boldsymbol{Y}=[\Gamma] \subseteq \bar{S}_{\varphi}$. On the other hand, the construction of $\psi$ guarantees that $\sup _{\beta} \widetilde{\psi}_{\beta}(x)=\infty$ for $x \notin \boldsymbol{Y}$ so $S_{\varphi} \subseteq \boldsymbol{Y}$ and $\bar{S}_{\varphi}=\boldsymbol{Y}$.

Given (C), there is a bounded generating set $\Gamma$ for $Y$ and $\nu=\nu_{r}$ is the required continuous norm on $Y^{*}$. Conversely, given $(\mathrm{E})$, let $B=\left\{\eta \in Y^{*}: \nu(\eta) \leqq 1\right\}$; the strong continuity of $\nu$ means $B$ is a neighborhood of 0 in $Y^{*}$ and so there exists a bounded set $\Gamma \subset Y$ such that $\Gamma^{0} \cong B$. Then $\nu_{\Gamma}$ is an everywhere finite continuous seminorm on $Y^{*}$ and, as $\nu_{\Gamma} \geqq \nu$ and $\nu$ is a norm, $\nu_{\Gamma}$ is a norm on $\boldsymbol{Y}^{*}$ and so $[\Gamma]=\boldsymbol{Y}$.

For convenience we state separately the result above in the case $\boldsymbol{X}=\boldsymbol{Y}$.

COROLLARY. The following conditions on a space $\boldsymbol{X}$ are equivalent:

(A) There exists $\varphi \in \Phi(X)$ such that $S_{\varphi}$ is dense.

(B) There is a bounded generating set for $\boldsymbol{X}$ (i.e., $\boldsymbol{X}$ is a $B G$ space).

(C) There exists a continuous norm ע on $X^{*}$. 
REmark. Clearly, if $\boldsymbol{X}$ is a Fréchet space the $\varphi$ of condition (A) may be taken to be countable.

Lemma. A Fréchet space $\boldsymbol{X}$ has a pre-compact generating set $K$ if and only if it is separable.

Proof. Suppose $\boldsymbol{X}$ is separable and $\left\{x_{1}, x_{2}, \cdots\right\}$ is a countable dense subset. Letting $\rho$ be any metric giving the topology on $X$, set $y_{n}=x_{n} /\left[1+\rho\left(0, x_{n}\right)\right]$ and $K=\left\{y_{n}\right\}$. Since $y_{n} \rightarrow 0, K$ is pre-compact. Since each $x_{n}$ is in the linear hull of $K$ and $\left\{x_{1}, x_{2}, \cdots\right\}$ is dense, $K$ is a generating set. Conversely, if $K$ is a pre-compact generating set then $\bar{K}$ (being a compact metric space) contains a countable dense subset $\left\{y_{1}, y_{2}, \cdots\right\}$; let $S$ be the set of all finite linear combinations of the $\left\{y_{n}\right\}$ with (complex) rational coefficients. Then $S$ is countable and, as $\left\{y_{1}, y_{2}, \cdots\right\}$ is dense generating set $\bar{K}, S$ is dense in $\boldsymbol{X}$ which is thus separable.

Since a pre-compact set must be bounded, any separable Fréchet space is a BG space and a positive partial answer to the question raised above is that, for a separable Fréchet space $X$, one can always choose $\varphi \in \Phi(X)$ so that $S_{\varphi}$ is dense; from the construction one can clearly arrange that $S_{\varphi}$ contain any specified countable set. We now collect some conditions under which a positive answer may be given to the question.

THeOREM 2. Any of the following is sufficient to ensure that $\boldsymbol{X}$ is a $B G$ space:
(A) $X$ is a Banach space.
(B) $\boldsymbol{X}$ is a separable Fréchet space.
(C) $X$ is a product of BG spaces.
(D) $\boldsymbol{X}$ is the image, under a continuous linear map, of a $B G$ space.
(E) $\boldsymbol{X}$ is a quotient of a product of Banach spaces.

Proof. (A) is trivial, (B) follows from the lemma above. (C) holds because the product of bounded subsets of the factors is bounded in a product space and the product of generating sets is a generating set. (D) holds because the image, under a continuous map, of a bounded set is bounded, of a dense set is dense. (E) follows immediately from (A), (C) and (D).

We now note that the final answer to the question: Does there always exist $\varphi \in \Phi$ such that $S_{\varphi}$ is dense? is negative even when restricted to Fréchet spaces. 
Example 2. Let $\Lambda=\left\{\lambda=\left[\lambda_{1}, \lambda_{2}, \cdots\right]: 1=\lambda_{1}<\lambda_{2}<\cdots ; \lambda_{n}\right.$ integers\} and let $\boldsymbol{H}$ be a Hilbert space big enough to contain an orthonormal set $\left\{a_{\lambda}: \lambda \in \Lambda\right\}$. Now let $\boldsymbol{X}=\prod_{1}^{\infty} \boldsymbol{H}_{n}$ with each $\boldsymbol{H}_{n}=\boldsymbol{H}$; the topology on $\boldsymbol{X}$ is determined by the sequence of semi-norms $\varphi=\left\{\varphi_{1}, \varphi_{2}, \cdots\right\}$; $\varphi_{n}(\boldsymbol{x})=\left\|\pi_{n} \boldsymbol{x}\right\|$ where $\pi_{n}: \boldsymbol{X} \rightarrow \boldsymbol{H}_{n}=\boldsymbol{H}$ is the canonical projection taking $\boldsymbol{x}=\left[x_{1}, x_{2}, \cdots\right] \in \boldsymbol{X}$ into $x_{n} \in \boldsymbol{H}$, the norm being that of $\boldsymbol{H}$. Then $\boldsymbol{X}$ is a Fréchet space; we observe that, by conditions (A) and (C) of Theorem 2, it is a BG space. For each $\lambda \in \Lambda$, set $b_{\lambda}=$ $\left[a_{\lambda}, \lambda_{2} a_{\lambda}, \lambda_{3} a_{\lambda}, \cdots\right] \in \boldsymbol{X}$ and let $\boldsymbol{Y}$ be the closed linear hull of $\left\{\boldsymbol{b}_{\lambda}: \lambda \in \Lambda\right\}$. We assert that the Fréchet space $\boldsymbol{Y}$ (we give $\boldsymbol{Y}$ the induced topology, determined by $\varphi$ ) contains no bounded generating sets, i.e., $\boldsymbol{Y}$ is not a $B G$ space.

Proof. We suppose, to the contrary, that $\Gamma$ is a bounded generating set for $Y$ (we may, and do, assume that $\Gamma$ is also closed, balanced, and convex) and proceed to show a contradiction. Boundedness of $\Gamma$ implies that each $\varphi_{n}$ is bounded on $\Gamma$ so $c_{n}=\sup \left\{\varphi_{n}(x): x \in \Gamma\right\}<\infty$ $(n=1,2, \cdots)$. Choose $\mu \in \Lambda$ such that $\sup _{n}\left\{\mu_{n} /\left(1+c_{n}\right)\right\}=\infty$; we show that $b_{\mu} \notin[\Gamma]$ so $\Gamma$ cannot be a generating set. Suppose, now, that

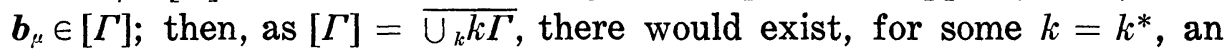
$\boldsymbol{x}^{(0)} \in k^{*} \Gamma$ such that $\phi_{1}\left(\boldsymbol{x}^{(0)}-\boldsymbol{b}_{\mu}\right)<1 / 4$. By the definition of $\boldsymbol{Y}$, there is, for each $n$, a linear combination $\boldsymbol{x}^{(n)}=\sum_{\lambda \in \Lambda} \beta_{\lambda} b_{\lambda}$ (a finite sum: $\beta_{\lambda}=$ $\beta_{\lambda}(n)$ vanishes for $\lambda$ not in some finite set, in general depending on n) such that both $\varphi_{1}\left(\boldsymbol{x}^{(0)}-\boldsymbol{x}^{(n)}\right)<1 / 4$ and $\varphi_{n}\left(\boldsymbol{x}^{(0)}-\boldsymbol{x}^{(n)}\right)<1$. Then $\varphi_{1}\left(\boldsymbol{x}^{(n)}-\boldsymbol{b}_{\mu}\right)<1 / 2$ so $1 / 4>\left\|\pi_{1}\left(\boldsymbol{x}^{(n)}-\boldsymbol{b}_{\mu}\right)\right\|^{2}=\Sigma_{\lambda \neq \mu}\left|\beta_{\lambda}\right|^{2}+\left|\beta_{\mu}-1\right|^{2}$ whence $\left|\beta_{\mu}\right|=\left|\beta_{\mu}(n)\right|>1 / 2$. Now $\varphi_{n}\left(x^{(0)}-\boldsymbol{x}^{(n)}\right)<1$ implies that

$$
\begin{aligned}
1> & \left\|\Sigma_{\lambda} \beta_{\lambda} \lambda_{n} a_{\lambda}-\pi_{n} \boldsymbol{x}^{(0)}\right\| \\
& \geqq\left\|\Sigma_{\lambda} \beta_{\lambda} \lambda_{n} a_{\lambda}\right\|-\left\|\boldsymbol{x}_{n}^{(0)}\right\| \geqq\left|\beta_{\mu}(n)\right| \mu_{n}-\varphi_{n}\left(\boldsymbol{x}^{(0)}\right) \geqq \mu_{n} / 2-k^{*} c_{n} .
\end{aligned}
$$

This, however, would imply that $\mu_{n} / 2 \leqq 1+k^{*} c_{n}$ so $\mu_{n} /\left(1+c_{n}\right) \leqq 2 k^{*}$ for $n=2,3, \cdots$ which contradicts the choice of the sequence $\mu$.

It is known that there may exist in Banach spaces, closed subspaces which are not the range of any continuous projection; considering $\boldsymbol{X}$ and $\boldsymbol{Y}$ of the example above in the light of condition (D) of Theorem 2 gives the following analogue.

COROLLARY. There exists Fréchet spaces (indeed, countable products of Banach spaces) containing closed subspaces which are not the range of any continuous linear operator on the space.

A number of open questions may be mentioned here.

(1) Ordering $\Phi$ by inclusion, let $\Phi_{0}$ be the set of all minimal 
families of semi-norms determining the topology on $\boldsymbol{X}$. In Example 1 we have $\psi \in \Phi_{0}$ and $S_{\psi}$ dense while $\varphi \notin \Phi_{0}$ and $S_{\varphi}$ not dense. If $\boldsymbol{X}$ is a BG space and $\varphi \in \Phi_{0}$, must $S_{\varphi}$ be dense?

(2) Call a space $\boldsymbol{X}$ a 'hereditary BG space' (HBG space) if every closed subspace is a BG space. Every Banach space and every separable Fréchet space is HBG. Are there any other HBG spaces? Is the product of two HBG spaces necessarily HBG? In particular, is the product of the spaces $\boldsymbol{X}$ of Example 1 and $\boldsymbol{H}$ of Example 2 an HBG space?

(3) Is every BG space a quotient of a product of Banach spaces? In particular, is every Fréchet BG space a quotient of a countable product of Banach spaces?

\section{REFERENCE}

1. L. Maté, On a semi-group of operators in Fréchet space, Doklady Akad. Nauk SSSR 142 (1962), 1247-1250.

(English translation in: Sov. Math. Doklady 3 (1962), 288-292.)

Received July 26, 1967.

WAyne State UNIVERSity

DETROIT, MichigaN 



\title{
PACIFIC JOURNAL OF MATHEMATICS
}

\author{
EDITORS
}

\author{
H. ROYDEN \\ Stanford University \\ Stanford, California
}

\author{
J. P. JANS \\ University of Washington \\ Seattle, Washington 98105
}

\section{J. DUGUNDJI}

Department of Mathematics University of Southern California Los Angeles, California 90007

\section{RICHARD ARENS}

University of California Los Angeles, California 90024

\section{ASSOCIATE EDITORS}

\author{
E. F. BECKENBACH
}

B. H. NEUMANN

F. WoLF

K. YosidA

\section{SUPPORTING INSTITUTIONS}

\author{
UNIVERSITY OF BRITISH COLUMBIA \\ CALIFORNIA INSTITUTE OF TECHNOLOGY \\ UNIVERSITY OF CALIFORNIA \\ MONTANA STATE UNIVERSITY \\ UNIVERSITY OF NEVADA \\ NEW MEXICO STATE UNIVERSITY \\ OREGON STATE UNIVERSITY \\ UNIVERSITY OF OREGON \\ OSAKA UNIVERSITY \\ UNIVERSITY OF SOUTHERN CALIFORNIA
}

\author{
STANFORD UNIVERSITY \\ UNIVERSITY OF TOKYO \\ UNIVERSITY OF UTAH \\ WASHINGTON STATE UNIVERSITY \\ UNIVERSITY OF WASHINGTON \\ AMERICAN MATHEMATICAL SOCIETY \\ CHEVRON RESEARCH CORPORATION \\ TRW SYSTEMS \\ NAVAL WEAPONS CENTER
}

Mathematical papers intended for publication in the Pacific Journal of Mathematics should be in typed form or offset-reproduced, double spaced with large margins. Underline Greek letters in red, German in green, and script in blue. The first paragraph or two must be capable of being used separately as a synopsis of the entire paper. It should not contain references to the bibliography. Manuscripts, in duplicate if possible, may be sent to any one of the four editors. All other communications to the editors should be addressed to the managing editor, Richard Arens, University of California, Los Angeles, California 90024.

Each author of each article receives 50 reprints free of charge; additional copies may be obtained at cost in multiples of 50 .

The Pacific Journal of Mathematics is published monthly. Effective with Volume 16 the price per volume (3 numbers) is $\$ 8.00$; single issues, $\$ 3.00$. Special price for current issues to individual faculty members of supporting institutions and to individual members of the American Mathematical Society: $\$ 4.00$ per volume; single issues $\$ 1.50$. Back numbers are available.

Subscriptions, orders for back numbers, and changes of address should be sent to Pacific Journal of Mathematics, 103 Highland Boulevard, Berkeley 8, California.

Printed at Kokusai Bunken Insatsusha (International Academic Printing Co., Ltd.), 7-17, Fujimi 2-chome, Chiyoda-ku, Tokyo, Japan.

PUBLISHED BY PACIFIC JOURNAL OF MATHEMATICS, A NON-PROFIT CORPORATION

The Supporting Institutions listed above contribute to the cost of publication of this Journal, but they are not owners of publishers and have no responsibility for its content or policies. 


\section{Pacific Journal of Mathematics}

Vol. 26, No. 2 December, 1968

Seymour Bachmuth and Horace Yomishi Mochizuki, Kostrikin's theorem on

Engel groups of prime power exponent ....................

Paul Richard Beesack and Krishna M. Das, Extensions of Opial's inequality ...................................... 215

John H. E. Cohn, Some quartic Diophantine equations .............. 233

H. P. Dikshit, Absolute $(C, 1) \cdot\left(N, p_{n}\right)$ summability of a Fourier series and its conjugate series ............................... 245

Raouf Doss, On measures with small transforms ................. 257

Charles L. Fefferman, $L_{p}$ spaces over finitely additive measures........ 265

Le Baron O. Ferguson, Uniform approximation by polynomials with integral coefficients. II...................................

Takashi Ito and Thomas I. Seidman, Bounded generators of linear

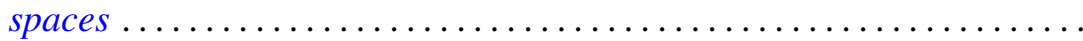

Masako Izumi and Shin-ichi Izumi, Nörlund summability of Fourier series ..........................................

Donald Gordon James, On Witt's theorem for unimodular quadratic

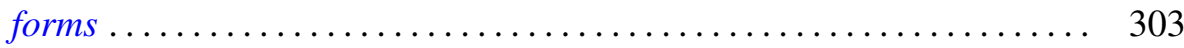

J. L. Kelley and Edwin Spanier, Euler characteristics .............. 317

Carl W. Kohls and Lawrence James Lardy, Some ring extensions with matrix

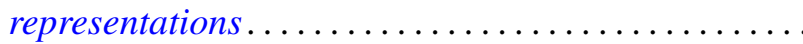

Ray Mines, III, A family of functors defined on generalized primary groups ....

Louise Arakelian Raphael, A characterization of integral operators on the space of Borel measurable functions bounded with respect to a weight function....

Charles Albert Ryavec, The addition of residue classes modulo $n .$.

H. M. (Hari Mohan) Srivastava, Fractional integration and inversion formulae associated with the generalized Whittaker transform ...

Edgar Lee Stout, The second Cousin problem with bounded data ...

Donald Curtis Taylor, A generalized Fatou theorem for Banach

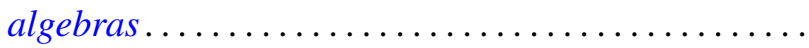

Bui An Ton, Boundary value problems for elliptic convolution equations of Wiener-Hopf type in a bounded region...

Philip C. Tonne, Bounded series and Hausdorff matrices for absolutely convergent sequences... 